\title{
Higher spins, quadratic forms and amplitudes
}

\section{Sudarshan Ananth, Chetan Pandey and Saurabh Pant}

Indian Institute of Science Education and Research, Pune 411008, India

E-mail: ananth@iiserpune.ac.in, chetan.pandey@students.iiserpune.ac.in, pant.saurabh@students.iiserpune.ac.in

ABSTRACT: The light-cone Hamiltonians for spin 1 and spin 2 fields, describing both the pure and the maximally supersymmetric theories, may be expressed as quadratic forms. In this paper, we show that this feature extends to light-cone higher spin theories. To first order in the coupling constant, we prove that the higher spin Hamiltonians, with and without supersymmetry, are quadratic forms. Scattering amplitude structures emerge naturally in this framework and we relate the momentum space vertex in a supersymmetric higher spin theory to the corresponding vertex in the $\mathcal{N}=4$ Yang-Mills theory.

KEYwords: Extended Supersymmetry, Superspaces, Scattering Amplitudes

ARXiv EPrint: 2005.10376 


\section{Contents}

1 Introduction 1

2 Cubic interaction vertices in higher spin theories $\quad 2$

2.1 Quadratic forms in higher spin theories - without supersymmetry 3

3 Cubic interaction vertices in supersymmetric higher spin theories 4

3.1 Quadratic forms in higher spin theories - with supersymmetry 6

3.1.1 Quadratic form $\neq$ anti-commutator 6

$\begin{array}{ll}3.1 .2 & \text { The proof } \\ \end{array}$

4 Amplitude structures in higher spin theories $\quad 10$

$\begin{array}{lll}4.1 & \text { The non-supersymmetric case } & 10\end{array}$

$\begin{array}{ll}4.2 \text { The supersymmetric case } & 10\end{array}$

$\begin{array}{ll}\text { A Superfield identities } & 12\end{array}$

\section{Introduction}

An interesting feature common to both Yang-Mills theory and gravity is that their lightcone Hamiltonians can be expressed as quadratic forms [1, 2]. This quadratic form structure appears exclusively in the pure and the maximally supersymmetric varieties $(\mathcal{N}=4$ superYang-Mills theory and $\mathcal{N}=8$ Supergravity $^{1}$ ). Simple structures like quadratic forms are interesting because they often signal the presence of a hidden symmetry or (and) produce considerable mathematical simplifications in the way we formulate these theories. This is in keeping with evidence that these theories - pure gravity and $\mathcal{N}=8$ supergravity for example - may possess hidden symmetries in four dimensions [3-6].

This paper focuses entirely on the light-cone Hamiltonian describing interacting higher spin fields [7-9], to first order in the coupling constant. Both the non-supersymmetric and supersymmetric theories, in four spacetime dimensions, are examined. We present the following three new results: (1) The light-cone Hamiltonian for higher spin fields without supersymmetry is a quadratic form, (2) Maximally supersymmetric higher spin theories also exhibit this quadratic form structure and (3) The momentum space vertex in a maximally supersymmetric higher spin theory is simply the $\mathcal{N}=4$ superYang-Mills theory vertex raised to the appropriate power.

The light-cone gauge has two key properties that make it particularly useful when studying scattering amplitude structures. First, it is not manifestly covariant - it has

\footnotetext{
${ }^{1}$ Even in the supersymmetric cases, this quadratic form is not a direct consequence of the fact that the Hamiltonian is the anti-commutator of the supersymmetry generators (see subsection 3.1.1).
} 
become increasingly clear that preserving manifest covariance obscures much of the simplicity we have come to associate with scattering amplitudes [10]. Second, the light-cone gauge focuses exclusively on physical degrees of freedom ensuring that spurious degrees of freedom do not hide the symmetries in a theory. These simplifications in amplitude structures are presented in section 4 .

\section{Cubic interaction vertices in higher spin theories}

We define light-cone co-ordinates in $(-,+,+,+)$ Minkowski space-time as

$$
x^{ \pm}=\frac{x^{0} \pm x^{3}}{\sqrt{2}}, \quad x=\frac{x^{1}+i x^{2}}{\sqrt{2}}, \quad \bar{x}=\frac{x^{1}-i x^{2}}{\sqrt{2}} .
$$

The corresponding derivatives are $\partial_{ \pm}, \bar{\partial}$ and $\partial$. In four spacetime dimensions, all massless fields have two physical degrees of freedom $\phi$ and $\bar{\phi}$ with $\lambda$ representing the positive value of the helicity of the field. $\partial_{+}=\frac{\partial \bar{\partial}}{\partial_{-}}$for a free theory, modified by corrections when interactions are switched on and $\frac{1}{\partial_{-}}$is defined following the prescription in [11].

The Hamiltonian for the free field theory is

$$
H \equiv \int d^{3} x \mathcal{H}=-\int d^{3} x \bar{\phi} \partial \bar{\partial} \phi,
$$

with the second equality being valid only for the free theory. We also write

$$
H \equiv \int d^{3} x \mathcal{H}=\int d^{3} x \partial_{-} \bar{\phi} \delta_{p^{-}} \phi,
$$

in terms of the time translation operator

$$
\delta_{p^{-}} \phi \equiv \partial_{+} \phi=\{\phi, \mathcal{H}\} .
$$

In the interacting case, $\delta_{p^{-}}$picks up corrections order by order in the coupling constant $g$.

Details regarding the derivation of light-cone cubic interaction vertices for higher spin theories are presented in $[7,8,12-15]$. The idea is to demand closure of the Poincare algebra which restricts and ultimately determines the light-cone Hamiltonian. The result is

$$
\delta_{p^{-}}^{g} \phi=g \sum_{n=0}^{\lambda}(-1)^{n}\left(\begin{array}{l}
\lambda \\
n
\end{array}\right) \partial_{-}^{\lambda-1}\left[\frac{\bar{\partial}^{\lambda-n}}{\partial_{-}^{\lambda-n}} \phi \frac{\bar{\partial}^{n}}{\partial_{-}^{n}} \phi\right],
$$

for even $\lambda$. For odd $\lambda$, algebra-closure requires an antisymmetric structure constant [16]

$$
\delta_{p^{-}}^{g} \phi^{a}=g f^{a b c} \sum_{n=0}^{\lambda}(-1)^{n}\left(\begin{array}{l}
\lambda \\
n
\end{array}\right) \partial_{-}^{\lambda-1}\left[\frac{\bar{\partial}^{\lambda-n}}{\partial_{-}^{\lambda-n}} \phi^{b} \frac{\bar{\partial}^{n}}{\partial_{-}^{n}} \phi^{c}\right] .
$$

From (2.3), the complete Hamiltonian to this order reads $[7,8]$

$$
H=\int d^{3} x\left(\partial \bar{\phi} \bar{\partial} \phi-g \sum_{n=0}^{\lambda}(-1)^{n}\left(\begin{array}{l}
\lambda \\
n
\end{array}\right) \bar{\phi} \partial_{-}^{\lambda}\left[\frac{\bar{\partial}^{\lambda-n}}{\partial_{-}^{\lambda-n}} \phi \frac{\bar{\partial}^{n}}{\partial_{-}^{n}} \phi\right]+\text { c.c. }\right),
$$

for even $\lambda$ and

$$
H=\int d^{3} x\left(\partial \bar{\phi}^{a} \bar{\partial} \phi^{a}-g f^{a b c} \sum_{n=0}^{\lambda}(-1)^{n}\left(\begin{array}{l}
\lambda \\
n
\end{array}\right) \bar{\phi}^{a} \partial_{-}^{\lambda}\left[\frac{\bar{\partial}^{\lambda-n}}{\partial_{-}^{\lambda-n}} \phi^{b} \frac{\bar{\partial}^{n}}{\partial_{-}^{n}} \phi^{c}\right]+c . c .\right),
$$

for odd $\lambda$. 


\subsection{Quadratic forms in higher spin theories — without supersymmetry}

In this section, we prove our first claim: that the Hamiltonians in (2.7) and (2.8) may be written as quadratic forms. Specifically, this means that the Hamiltonians have the following "whole square" form

$$
H=\int d^{3} x \overline{\mathcal{D}} \phi \mathcal{D} \bar{\phi},
$$

in terms of "covariant" derivatives. Covariance, specifically for $\lambda=1,2$ was demonstrated in $[1,2]$. We find (structure constants not shown explicitly)

$$
\begin{aligned}
& \mathcal{D} \bar{\phi}=\partial \bar{\phi}-2 g \sum_{n=0}^{\lambda-1}(-1)^{n}\left(\begin{array}{c}
\lambda-1 \\
n
\end{array}\right) \frac{\bar{\partial}^{n}}{\partial_{-}^{n+1}}\left[\frac{\bar{\partial}^{\lambda-n-1}}{\partial_{-}^{\lambda-n-1}} \phi \partial_{-}^{\lambda} \bar{\phi}\right] \\
& \overline{\mathcal{D}} \phi=\bar{\partial} \phi-2 g \sum_{n=0}^{\lambda-1}(-1)^{n}\left(\begin{array}{c}
\lambda-1 \\
n
\end{array}\right) \frac{\partial^{n}}{\partial_{-}^{n+1}}\left[\frac{\partial^{\lambda-n-1}}{\partial_{-}^{\lambda-n-1}} \bar{\phi} \partial_{-}^{\lambda} \phi\right]
\end{aligned}
$$

which reproduce the correct results for both Yang-Mills theory and gravity.

From these definitions, it follows that (2.9) correctly produces the kinetic term in the Hamiltonians. To prove its equivalence to (2.7) and (2.8), we therefore focus on the $O(g)$ contributions from (2.9). These are (measure not shown explicitly)

$$
-2 g \sum_{n=0}^{\lambda-1}(-1)^{n}\left(\begin{array}{c}
\lambda-1 \\
n
\end{array}\right)\left[\bar{\partial} \phi \frac{\bar{\partial}^{n}}{\partial_{-}^{n+1}}\left(\frac{\bar{\partial}^{\lambda-n-1}}{\partial_{-}^{\lambda-n-1}} \phi \partial_{-}^{\lambda} \bar{\phi}\right)\right],
$$

and its complex conjugate. We partially integrate this expression to obtain

$$
-2 g \sum_{n=0}^{\lambda-1}(-1)^{\lambda+n+1}\left(\begin{array}{c}
\lambda-1 \\
n
\end{array}\right) \bar{\phi} \partial_{-}^{\lambda}\left[\frac{\bar{\partial}^{n+1}}{\partial_{-}^{n+1}} \phi \frac{\bar{\partial}^{\lambda-n-1}}{\partial_{-}^{\lambda-n-1}} \phi\right] .
$$

We split (2.13) into two halves $P$ and $Q$. In $P$, we shift $n \rightarrow \lambda-n-1$ and invoke the identity

$$
\left(\begin{array}{c}
\lambda-1 \\
n
\end{array}\right)=\left(\begin{array}{c}
\lambda-1 \\
\lambda-1-n
\end{array}\right),
$$

which yields

$$
P=-g \sum_{n=0}^{\lambda-1}(-1)^{n}\left(\begin{array}{c}
\lambda-1 \\
n
\end{array}\right) \bar{\phi} \partial_{-}{ }^{\lambda}\left[\frac{\bar{\partial}^{\lambda-n}}{\partial_{-}^{\lambda-n}} \phi \frac{\bar{\partial}^{n}}{\partial_{-}^{n}} \phi\right] .
$$

In the other half $Q$ we shift $n \rightarrow n-1$ to obtain

$$
Q=-g \sum_{n=1}^{\lambda}(-1)^{\lambda+n}\left(\begin{array}{l}
\lambda-1 \\
n-1
\end{array}\right) \bar{\phi} \partial_{-}^{\lambda}\left[\frac{\bar{\partial}^{n}}{\partial_{-}^{n}} \phi \frac{\bar{\partial}^{\lambda-n}}{\partial_{-}^{\lambda-n}} \phi\right] .
$$


We then have

$$
\begin{aligned}
H=P+Q= & \left\{-g \sum_{n=0}^{\lambda-1}(-1)^{n}\left(\begin{array}{c}
\lambda-1 \\
n
\end{array}\right) \bar{\phi} \partial_{-}{ }^{\lambda}\left[\frac{\bar{\partial}^{\lambda-n}}{\partial_{-}^{\lambda-n}} \phi \frac{\bar{\partial}^{n}}{\partial_{-}^{n}} \phi\right]\right. \\
& \left.-g \sum_{n=1}^{\lambda}(-1)^{\lambda+n}\left(\begin{array}{c}
\lambda-1 \\
n-1
\end{array}\right) \bar{\phi} \partial_{-}^{\lambda}\left[\frac{\bar{\partial}^{n}}{\partial_{-}^{n}} \phi \frac{\bar{\partial}^{\lambda-n}}{\partial_{-}^{\lambda-n}} \phi\right]\right\}, \\
= & -g \sum_{n=0}^{\lambda}(-1)^{n}\left(\left[\left(\begin{array}{c}
\lambda-1 \\
n
\end{array}\right)+\left(\begin{array}{c}
\lambda-1 \\
n-1
\end{array}\right)\right] \bar{\phi} \partial_{-}^{\lambda}\left[\frac{\bar{\partial}^{\lambda-n}}{\partial_{-}^{\lambda-n}} \phi \frac{\bar{\partial}^{n}}{\partial_{-}^{n}} \phi\right]\right) .
\end{aligned}
$$

Using the Pascal triangle property

$$
\left(\begin{array}{c}
\lambda-1 \\
n
\end{array}\right)+\left(\begin{array}{l}
\lambda-1 \\
n-1
\end{array}\right)=\left(\begin{array}{l}
\lambda \\
n
\end{array}\right)
$$

this is

$$
H=P+Q=-g \sum_{n=0}^{\lambda}(-1)^{n}\left(\begin{array}{l}
\lambda \\
n
\end{array}\right) \bar{\phi} \partial_{-}^{\lambda}\left[\frac{\bar{\partial}^{\lambda-n}}{\partial_{-}^{\lambda-n}} \phi \frac{\bar{\partial}^{n}}{\partial_{-}^{n}} \phi\right],
$$

reproducing the structures in (2.7), (2.8) and confirming that the higher spin Hamiltonians are indeed quadratic forms.

\section{Cubic interaction vertices in supersymmetric higher spin theories}

We now move to theories of arbitrary integer spin with supersymmetry. We work in lightcone superspace where the supersymmetry generators are of two varieties [9]. Realized on Grassmann parameters $\theta^{m}$ and their conjugates $\bar{\theta}_{m}$, the kinematical generators are

$$
q^{m}=-\frac{\partial}{\partial \bar{\theta}_{m}}-\frac{i}{\sqrt{2}} \theta^{m} \partial_{-}, \quad \bar{q}_{n}=\frac{\partial}{\partial \theta^{n}}+\frac{i}{\sqrt{2}} \bar{\theta}_{n} \partial_{-},
$$

and satisfy

$$
\left\{q^{m}, \bar{q}_{n}\right\}=-\sqrt{2} \delta_{n}^{m} p^{+} .
$$

The dynamical generators satisfy

$$
\left\{Q^{m}, \bar{Q}_{n}\right\}=\delta_{n}^{m} p^{-}
$$

thus representing "square roots" of the light-cone Hamiltonian. At lowest order, they are

$$
Q^{m}=-\frac{\bar{\partial}}{\partial_{-}} q^{m}, \quad \bar{Q}_{m}=-\frac{\partial}{\partial_{-}} \bar{q}_{m} .
$$

These expression pick up corrections order by order in the coupling constant in an interacting theory. We introduce superspace derivatives $d^{m}$ and $\bar{d}_{n}$

$$
d^{m}=-\frac{\partial}{\partial \bar{\theta}_{m}}+\frac{i}{\sqrt{2}} \theta^{m} \partial_{-}, \quad \bar{d}_{n}=\frac{\partial}{\partial \theta^{n}}-\frac{i}{\sqrt{2}} \bar{\theta}_{n} \partial_{-},
$$


which anti-commute with the supersymmetry generators and satisfy

$$
\left\{d^{m}, \overline{d_{n}}\right\}=i \sqrt{2} \delta_{n}^{m} \partial_{-} .
$$

We use $\Phi$ to denote a superfield and impose a "chirality" condition on it so

$$
\mathrm{d}^{m} \Phi(x, \theta, \bar{\theta})=0 .
$$

For maximally extended supermultiplets, we have the additional "inside-out" constraint

$$
\bar{\Phi}(x, \theta, \bar{\theta})=\frac{1}{2^{N / 4} N !} \frac{\overline{\mathrm{d}}^{N}}{\partial_{-}^{N / 2}} \Phi(x, \theta, \bar{\theta}),
$$

with

$$
N=4 \lambda,
$$

in this section and the following one. The Hamiltonian in this formalism is

$$
H \equiv \int d^{3} x \mathrm{~d}^{N} \theta \mathrm{d}^{N} \bar{\theta} \mathcal{H}=\int d^{3} x \mathrm{~d}^{N} \theta \mathrm{d}^{N} \bar{\theta} \partial_{-} \bar{\Phi} \delta_{p^{-}} \Phi .
$$

Algebra-closure now involves the larger superPoincaré algebra [17] and yields [9]

$$
Q^{m} \bar{\Phi}=-\frac{\bar{\partial}}{\partial_{-}} q^{m} \bar{\Phi}-2 g \sum_{n=0}^{\lambda-1}(-1)^{n}\left(\begin{array}{c}
\lambda-1 \\
n
\end{array}\right) \frac{1}{\partial_{-}}\left(\partial^{(\lambda-1-n)} \partial_{-}^{n} d^{m} \bar{\Phi} \partial_{-}^{(\lambda-n)} \partial^{n} \bar{\Phi}\right)+\mathcal{O}\left(g^{2}\right)
$$

for the dynamical supersymmetry generator

$$
\delta_{p^{-}}^{g} \Phi=g \sum_{n=0}^{\lambda}(-1)^{n}\left(\begin{array}{l}
\lambda \\
n
\end{array}\right) \frac{1}{\partial_{-}}\left[\bar{\partial}^{(\lambda-n)} \partial_{-}^{n} \Phi \bar{\partial}^{n} \partial_{-}^{(\lambda-n)} \Phi\right],
$$

for even $\lambda$ and

$$
\delta_{p^{-}}^{g} \Phi^{a}=g f^{a b c} \sum_{n=0}^{\lambda}(-1)^{n}\left(\begin{array}{l}
\lambda \\
n
\end{array}\right) \frac{1}{\partial_{-}}\left[\bar{\partial}^{(\lambda-n)} \partial_{-}^{n} \Phi^{b} \bar{\partial}^{n} \partial_{-}^{(\lambda-n)} \Phi^{c}\right],
$$

for odd $\lambda$. The corresponding Hamiltonians being

$$
\begin{aligned}
H= & \int \mathrm{d}^{3} x \mathrm{~d}^{N} \theta \mathrm{d}^{N} \bar{\theta}\left\{\frac{1}{2} \partial \bar{\Phi} \frac{\bar{\partial}}{\partial_{-}^{N / 2}} \Phi\right. \\
& \left.-\frac{1}{3} g\left(\frac{1}{\partial_{-}^{N / 2}} \bar{\Phi} \sum_{n=0}^{\lambda}(-1)^{n}\left(\begin{array}{l}
\lambda \\
n
\end{array}\right)\left[\bar{\partial}^{(\lambda-n)} \partial_{-}^{n} \Phi \bar{\partial}^{n} \partial_{-}^{(\lambda-n)} \Phi\right]+\text { c.c. }\right)+\mathcal{O}\left(g^{2}\right)\right\},
\end{aligned}
$$

for even $\lambda$ and

$$
\begin{aligned}
H= & \int \mathrm{d}^{3} x \mathrm{~d}^{N} \theta \mathrm{d}^{N} \bar{\theta}\left\{\frac{1}{2} \partial \bar{\Phi}^{a} \frac{\bar{\partial}}{\partial_{-}^{N / 2}} \Phi^{a}\right. \\
& \left.-\frac{1}{3} g f^{a b c}\left[\frac{1}{\partial_{-}^{N / 2}} \bar{\Phi}^{a} \sum_{n=0}^{\lambda}(-1)^{n}\left(\begin{array}{l}
\lambda \\
n
\end{array}\right)\left[\bar{\partial}^{(\lambda-n)} \partial_{-}^{n} \Phi^{b} \bar{\partial}^{n} \partial_{-}^{(\lambda-n)} \Phi^{c}\right]+c . c .\right]+\mathcal{O}\left(g^{2}\right)\right\}
\end{aligned}
$$

for odd $\lambda$. 


\subsection{Quadratic forms in higher spin theories — with supersymmetry}

We now prove, to first order in the coupling constant, that the light-cone Hamiltonians in (3.14) and (3.15) are quadratic forms. The specific claim is that these Hamiltonians can be written as follows.

$$
H=\frac{2}{N \sqrt{2}}\left(\mathcal{W}_{m}, \mathcal{W}_{m}\right)
$$

with

$$
(\Phi, \Xi) \equiv 2 i \int d^{3} x d^{N} \theta d^{N} \bar{\theta} \bar{\Phi} \frac{1}{\partial_{-}^{2 \lambda-1}} \Xi
$$

\subsubsection{Quadratic form $\neq$ anti-commutator}

Before proving (3.16), we explain how the "quadratic form" concept differs from the idea of writing the Hamiltonian as the anti-commutator of dynamical supersymmetries. This is best illustrated by restricting ourselves to the lowest order dynamical supersymmetry generators. Start with the simple choice ${ }^{2}$

$$
\overline{\mathcal{W}}^{m}=Q^{m} \bar{\Phi},
$$

where keep just the $g=0$ piece in (3.11). The claim in (3.16) is that the Hamiltonian reads (measure, integrals and constants are suppressed and a factor of 2 included for convenience)

$$
H=\left(\mathcal{W}_{m}, \mathcal{W}_{m}\right)=2 Q^{m} \bar{\Phi} \frac{1}{\partial_{-}^{2 \lambda-1}} \bar{Q}_{m} \Phi
$$

The step above is valid in any theory with supersymmetry. However, exclusive to maximally supersymmetric theories is the inside-out relation in (3.8) which we now invoke. Write (3.19) as two halves and apply the inside-out relation only to the second expression to obtain

$$
H=Q^{m} \bar{\Phi} \frac{1}{\partial_{-}^{2 \lambda-1}} \bar{Q}_{m} \Phi+Q^{m} \frac{1}{\partial_{-}^{2 \lambda}} \Phi \partial_{-} \bar{Q}_{m} \bar{\Phi} .
$$

Rewriting the second term this way is not possible in theories with less than maximal supersymmetry.

In (3.20), integrate the $Q$ in the first expression to the right and the $\bar{Q}$ in the second expression to the left to obtain

$$
H=-\bar{\Phi} \frac{1}{\partial_{-}^{2 \lambda-1}} Q^{m} \bar{Q}_{m} \Phi+\bar{Q}_{m} Q^{m} \frac{1}{\partial_{-}^{2 \lambda}} \Phi \partial_{-} \bar{\Phi} .
$$

Finally, in the second expression, integrate the $\partial_{-}$once leaving us with

$$
H=-\bar{\Phi} \frac{1}{\partial_{-}^{2 \lambda-1}} Q^{m} \bar{Q}_{m} \Phi-\bar{Q}_{m} Q^{m} \frac{1}{\partial_{-}^{2 \lambda-1}} \Phi \bar{\Phi},
$$

which is equivalent to

$$
H=-\bar{\Phi} \frac{1}{\partial_{-}^{2 \lambda-1}}\left\{Q^{m}, \bar{Q}_{m}\right\} \Phi,
$$

\footnotetext{
${ }^{2}$ This choice preserves chirality, ie. acting with a $\bar{d}$ on (3.18) yields 0 .
} 
known to be a true statement. Thus a quadratic form structure as in (3.16) can only appear in maximally supersymmetric theories, where it is equivalent to (3.23) at the lowest order.

It is important to remember that the $Q$ in (3.20) is non-linearly realized on the superfields beyond the lowest order and cannot be integrated as in (3.21) if we were to include higher order contributions to (3.18).

\subsubsection{The proof}

We have already identified $\mathcal{W}$ with the action of the dynamical supersymmetry on the superfield in (3.18). Accordingly, including the first order contributions we have

$$
\overline{\mathcal{W}}^{m}=-\frac{\bar{\partial}}{\partial_{-}} q^{m} \bar{\Phi}-2 g \sum_{k=0}^{\lambda-1}(-1)^{k}\left(\begin{array}{c}
\lambda-1 \\
k
\end{array}\right) \frac{1}{\partial_{-}}\left(\partial^{(\lambda-1-k)} \partial_{-}{ }^{(k)} d^{m} \bar{\Phi} \partial_{-}^{(\lambda-k)} \partial^{k} \bar{\Phi}\right)+\mathcal{O}\left(g^{2}\right)
$$

with the appropriate structure constants for odd $\lambda$. The kinetic contribution from (3.16) is trivial. At cubic order, the Hamiltonian involves terms of the form

$$
H=\frac{8 i}{N \sqrt{2}} g \frac{\bar{\partial}}{\partial_{-}^{2 \lambda+1}} q^{m} \bar{\Phi} \sum_{k=0}^{\lambda-1}(-1)^{k}\left(\begin{array}{c}
\lambda-1 \\
k
\end{array}\right)\left(\bar{\partial}^{(\lambda-1-k)} \partial_{-}^{k} \bar{d}_{m} \Phi \partial_{-}^{(\lambda-k)} \bar{\partial}^{k} \Phi\right) .
$$

We use $q^{m} X=-i \sqrt{2} \theta^{m} \partial_{-} X$ where $X$ is any chiral combination of superfields [17], and $\theta^{m} \bar{d}_{m}=N \theta^{1} \bar{d}_{1}$ to simplify this to

$$
H=8 g \frac{\bar{\partial}}{\partial_{-}^{2 \lambda}} \theta^{1} \bar{\Phi} \sum_{k=0}^{\lambda-1}(-1)^{k}\left(\begin{array}{c}
\lambda-1 \\
k
\end{array}\right)\left(\bar{\partial}^{(\lambda-1-k)} \partial_{-}^{k} \bar{d}_{1} \Phi \partial_{-}^{(\lambda-k)} \bar{\partial}^{k} \Phi\right) .
$$

Equation (3.26) is our starting point and we will rewrite it in two different ways. The first rewriting involves integrating the $\bar{\partial}$ from the first superfield to produce two terms, $I$ and $J$

$$
\begin{aligned}
H= & -8 g \frac{1}{\partial_{-}^{2 \lambda}} \theta^{1} \bar{\Phi} \sum_{k=0}^{\lambda-1}(-1)^{k}\left(\begin{array}{c}
\lambda-1 \\
k
\end{array}\right)\left(\bar{\partial}^{(\lambda-k)} \partial_{-}^{k} \bar{d}_{1} \Phi \partial_{-}^{(\lambda-k)} \bar{\partial}^{k} \Phi\right) \\
& -8 g \frac{1}{\partial_{-}^{2 \lambda}} \theta^{1} \bar{\Phi} \sum_{k=0}^{\lambda-1}(-1)^{k}\left(\begin{array}{c}
\lambda-1 \\
k
\end{array}\right)\left(\bar{\partial}^{(\lambda-1-k)} \partial_{-}^{k} \bar{d}_{1} \Phi \partial_{-}^{(\lambda-k)} \bar{\partial}^{(k+1)} \Phi\right) \\
= & I+J
\end{aligned}
$$

This form for the Hamiltonian will be used later in this subsection.

We now rewrite (3.26) in a second manner (terms in blue survive the manipulations described below). The first step is to partially integrate the $\bar{d}_{1}$ in (3.26), to obtain two terms

$$
\begin{aligned}
H= & +8 g \frac{\bar{\partial}}{\partial_{-}^{2 \lambda}} \bar{\Phi} \sum_{k=0}^{\lambda-1}(-1)^{k}\left(\begin{array}{c}
\lambda-1 \\
k
\end{array}\right)\left(\bar{\partial}^{(\lambda-1-k)} \partial_{-}^{k} \Phi \partial_{-}^{(\lambda-k)} \bar{\partial}^{k} \Phi\right) \\
& -8 g \frac{\bar{\partial}}{\partial_{-}^{2 \lambda}} \bar{\Phi} \sum_{k=0}^{\lambda-1}(-1)^{k}\left(\begin{array}{c}
\lambda-1 \\
k
\end{array}\right)\left(\bar{\partial}^{(\lambda-1-k)} \partial_{-}^{k} \Phi \partial_{-}^{(\lambda-k)} \bar{\partial}^{k} \theta^{1} \bar{d}_{1} \Phi\right)
\end{aligned}
$$


In (3.29), a $\partial_{-}$is integrated away from the last superfield to yield

$$
\begin{aligned}
& +8 g \frac{\bar{\partial}}{\partial_{-}^{2 \lambda}} \bar{\Phi} \sum_{k=0}^{\lambda-1}(-1)^{k}\left(\begin{array}{c}
\lambda-1 \\
k
\end{array}\right)\left(\bar{\partial}^{(\lambda-1-k)} \partial_{-}^{(k+1)} \Phi \partial_{-}^{(\lambda-k-1)} \bar{\partial}^{k} \theta^{1} \bar{d}_{1} \Phi\right) \\
& +8 g \frac{\bar{\partial}}{\partial_{-}^{2 \lambda-1}} \bar{\Phi} \sum_{k=0}^{\lambda-1}(-1)^{k}\left(\begin{array}{c}
\lambda-1 \\
k
\end{array}\right)\left(\bar{\partial}^{(\lambda-1-k)} \partial_{-}^{k} \Phi \partial_{-}^{(\lambda-k-1)} \bar{\partial}^{k} \theta^{1} \bar{d}_{1} \Phi\right)
\end{aligned}
$$

Equation (3.30) is the negative of (3.26) since

$$
\left(\begin{array}{c}
\lambda-1 \\
k
\end{array}\right)=\left(\begin{array}{c}
\lambda-1 \\
\lambda-1-k
\end{array}\right)
$$

allowing us to combine it with (3.26) producing a factor of two. Equation (3.31) can be simplifed, using integrations and the inside-out constraint, to

$$
\begin{aligned}
& +4 g \bar{\partial} \partial_{-} \Phi \sum_{k=0}^{\lambda-1}(-1)^{k}\left(\begin{array}{c}
\lambda-1 \\
k
\end{array}\right)\left(\bar{\partial}^{(\lambda-1-k)} \partial_{-}^{k} \frac{1}{\partial_{-}^{2 \lambda}} \bar{\Phi} \partial_{-}^{(\lambda-k-1)} \bar{\partial}^{k} \theta^{1} \bar{d}_{1} \Phi\right) \\
& -4 g \bar{\partial} \partial_{-} \bar{d}_{1} \Phi \sum_{k=0}^{\lambda-1}(-1)^{k}\left(\begin{array}{c}
\lambda-1 \\
k
\end{array}\right)\left(\bar{\partial}^{(\lambda-1-k)} \partial_{-}^{k} \frac{1}{\partial_{-}^{2 \lambda}} \bar{\Phi} \partial_{-}^{(\lambda-k-1)} \bar{\partial}^{k} \theta^{1} \Phi\right) \\
& -4 g \bar{\partial} \bar{d}_{1} \Phi \sum_{k=0}^{\lambda-1}(-1)^{k}\left(\begin{array}{c}
\lambda-1 \\
k
\end{array}\right)\left(\bar{\partial}^{(\lambda-1-k)} \partial_{-}^{k} \frac{1}{\partial_{-}^{2 \lambda-1}} \bar{\Phi} \partial_{-}^{(\lambda-k-1)} \bar{\partial}^{k} \theta^{1} \Phi\right)
\end{aligned}
$$

We simplify (3.33) by integrating the $\bar{d}_{1}$ from the first superfield, yielding

$$
\begin{aligned}
& +4 g \bar{\partial} \partial_{-} \Phi \sum_{k=0}^{\lambda-1}(-1)^{k}\left(\begin{array}{c}
\lambda-1 \\
k
\end{array}\right)\left(\bar{\partial}^{(\lambda-1-k)} \partial_{-}^{k} \frac{1}{\partial_{-}^{2 \lambda}} \bar{\Phi} \partial_{-}^{(\lambda-k-1)} \bar{\partial}^{k} \Phi\right) \\
& -4 g \bar{\partial} \partial_{-} \Phi \sum_{k=0}^{\lambda-1}(-1)^{k}\left(\begin{array}{c}
\lambda-1 \\
k
\end{array}\right)\left(\bar{\partial}^{(\lambda-1-k)} \partial_{-}^{k} \frac{1}{\partial_{-}^{2 \lambda}} \bar{\Phi} \partial_{-}^{(\lambda-k-1)} \bar{\partial}^{k} \theta^{1} \bar{d}_{1} \Phi\right)
\end{aligned}
$$

and find that (3.36) cancels against (3.32). Integrating the chiral derivative and using partial integrations simplifies (3.34) to

$$
\begin{aligned}
& +4 g \bar{\partial} \Phi \sum_{k=0}^{\lambda-1}(-1)^{k}\left(\begin{array}{c}
\lambda-1 \\
k
\end{array}\right)\left(\bar{\partial}^{(\lambda-1-k)} \partial_{-}^{k} \frac{1}{\partial_{-}^{2 \lambda-1}} \bar{\Phi} \partial_{-}^{(\lambda-k-1)} \bar{\partial}^{k} \Phi\right) \\
& -4 g \frac{\bar{\partial}}{\partial_{-}^{2 \lambda-1}} \bar{\Phi} \sum_{k=0}^{\lambda-1}(-1)^{k}\left(\begin{array}{c}
\lambda-1 \\
k
\end{array}\right) \bar{\partial}^{(\lambda-1-k)} \partial_{-}^{k}\left(\Phi \partial_{-}^{(\lambda-k-1)} \bar{\partial}^{k} \theta^{1} \bar{d}_{1} \Phi\right) \\
& +4 g \frac{\bar{\partial}}{\partial_{-}^{2 \lambda-1}} \bar{\Phi} \sum_{k=0}^{\lambda-1}(-1)^{k}\left(\begin{array}{c}
\lambda-1 \\
k
\end{array}\right) \bar{\partial}^{(\lambda-2-k)} \partial_{-}^{k}\left(\Phi \partial_{-}^{(\lambda-k-1)} \bar{\partial}^{(k+1)} \theta^{1} \bar{d}_{1} \Phi\right)
\end{aligned}
$$

Note that (3.38) is the negative of (3.31) using the identity (A.2) from appendix A (these terms combine with a factor of two). Partial integrations of $\partial_{-}$and $\bar{\partial}$ in (3.39) followed by 
the use of a similar identity (A.3) yields

$$
\begin{aligned}
& +2 g \frac{1}{\partial_{-}^{2 \lambda}} \bar{\Phi} \sum_{k=0}^{\lambda-1}(-1)^{k}\left(\begin{array}{c}
\lambda-1 \\
k
\end{array}\right)\left(\bar{\partial}^{\lambda-1-k} \partial_{-}^{k} \Phi \partial_{-}^{(\lambda-k)} \bar{\partial}^{(k+1)} \theta^{1} \bar{d}_{1} \Phi\right), \\
& +2 g \frac{1}{\partial_{-}^{2 \lambda}} \bar{\Phi} \sum_{k=0}^{\lambda-1}(-1)^{k}\left(\begin{array}{c}
\lambda-1 \\
k
\end{array}\right)\left(\bar{\partial}^{\lambda-1-k} \partial_{-}^{(k+1)} \Phi \partial_{-}^{(\lambda-k-1)} \bar{\partial}^{(k+1)} \theta^{1} \bar{d}_{1} \Phi\right) .
\end{aligned}
$$

This completes the second re-writing of (3.26).

We have now rewritten (3.26) in two ways. We now subtract $\frac{1}{4}$ times the first form in (3.27), written in green, from the second form of (3.26), in blue. Thus

$$
\begin{aligned}
H-\frac{1}{4} H= & +4 g \frac{\bar{\partial}}{\partial_{-}^{2 \lambda}} \bar{\Phi} \sum_{k=0}^{\lambda-1}(-1)^{k}\left(\begin{array}{c}
\lambda-1 \\
k
\end{array}\right)\left(\bar{\partial}^{(\lambda-1-k)} \partial_{-}^{k} \Phi \partial_{-}^{(\lambda-k)} \bar{\partial}^{k} \Phi\right) \\
& +2 g \bar{\partial} \partial_{-} \Phi \sum_{k=0}^{\lambda-1}(-1)^{k}\left(\begin{array}{c}
\lambda-1 \\
k
\end{array}\right)\left(\bar{\partial}^{(\lambda-1-k)} \partial_{-}^{k} \frac{1}{\partial_{-}^{2 \lambda}} \bar{\Phi} \partial_{-}^{(\lambda-k-1)} \bar{\partial}^{k} \Phi\right) \\
& +2 g \bar{\partial} \Phi \sum_{k=0}^{\lambda-1}(-1)^{k}\left(\begin{array}{c}
\lambda-1 \\
k
\end{array}\right)\left(\bar{\partial}^{(\lambda-1-k)} \partial_{-}^{k} \frac{1}{\partial_{-}^{2 \lambda-1}} \bar{\Phi} \partial_{-}^{(\lambda-k-1)} \bar{\partial}^{k} \Phi\right) \\
& +2 g \frac{1}{\partial_{-}^{2 \lambda}} \bar{\Phi} \sum_{k=0}^{\lambda-1}(-1)^{k}\left(\begin{array}{c}
\lambda-1 \\
k
\end{array}\right)\left(\bar{\partial}^{\lambda-1-k} \partial_{-}^{k} \Phi \partial_{-}^{(\lambda-k)} \bar{\partial}^{(k+1)} \theta^{1} \bar{d}_{1} \Phi\right) \\
& -2 g \frac{1}{\partial_{-}^{2 \lambda}} \theta^{1} \bar{\Phi} \sum_{k=0}^{\lambda-1}(-1)^{k}\left(\begin{array}{c}
\lambda-1 \\
k
\end{array}\right)\left(\bar{\partial}^{(\lambda-1-k)} \partial_{-}^{k} \bar{d}_{1} \Phi \partial_{-}^{(\lambda-k)} \bar{\partial}^{(k+1)} \Phi\right) \\
& +2 g \frac{1}{\partial_{-}^{2 \lambda}} \theta^{1} \bar{\Phi} \sum_{k=0}^{\lambda-1}(-1)^{k}\left(\begin{array}{c}
\lambda-1 \\
k
\end{array}\right)\left(\bar{\partial}^{(\lambda-k)} \partial_{-}^{k} \bar{d}_{1} \Phi \partial_{-}^{(\lambda-k)} \bar{\partial}^{k} \Phi\right) \\
& +2 g \frac{1}{\partial_{-}^{2 \lambda}} \bar{\Phi} \sum_{k=0}^{\lambda-1}(-1)^{k}\left(\begin{array}{c}
\lambda-1 \\
k
\end{array}\right)\left(\bar{\partial}^{\lambda-1-k} \partial_{-}^{(k+1)} \Phi \partial_{-}^{(\lambda-k-1)} \bar{\partial}^{(k+1)} \theta^{1} \bar{d}_{1} \Phi\right)
\end{aligned}
$$

Terms (3.45) and (3.46) combine into a single term (chain rule for $\bar{d}_{1}$ ). Terms (3.47) and (3.48) cancel due to the combinatorial identity. Integration of $\partial_{-}$in (3.43) produces two terms, one of which cancels (3.44). The inside-out relation simplifies the other term to

$$
-2 g \frac{\bar{\partial}}{\partial_{-}^{2 \lambda}} \bar{\Phi} \sum_{k=0}^{\lambda-1}(-1)^{k}\left(\begin{array}{c}
\lambda-1 \\
k
\end{array}\right)\left(\bar{\partial}^{(\lambda-1-k)} \partial_{-}^{k} \Phi \partial_{-}^{(\lambda-k)} \bar{\partial}^{k} \Phi\right),
$$

which combines with (3.42). In this combination, we integrate the $\bar{\partial}$ to obtain two terms

$$
\begin{aligned}
& -2 g \frac{1}{\partial_{-}^{2 \lambda}} \bar{\Phi} \sum_{k=0}^{\lambda-1}(-1)^{k}\left(\begin{array}{c}
\lambda-1 \\
k
\end{array}\right)\left(\bar{\partial}^{(\lambda-k)} \partial_{-}^{k} \Phi \partial_{-}^{(\lambda-k)} \bar{\partial}^{k} \Phi\right) \\
& -2 g \frac{1}{\partial_{-}^{2 \lambda}} \bar{\Phi} \sum_{k=0}^{\lambda-1}(-1)^{k}\left(\begin{array}{c}
\lambda-1 \\
k
\end{array}\right)\left(\bar{\partial}^{(\lambda-1-k)} \partial_{-}^{k} \Phi \partial_{-}^{(\lambda-k)} \bar{\partial}^{(k+1)} \Phi\right)
\end{aligned}
$$


with (3.51) canceling the (3.45)-(3.46) combine leaving us with (3.50). We simplify (3.50) as follows.

$$
\begin{aligned}
H & =-\frac{8}{3} g \frac{1}{\partial_{-}^{2 \lambda}} \bar{\Phi} \sum_{k=0}^{\lambda-1}(-1)^{k}\left(\begin{array}{c}
\lambda-1 \\
k
\end{array}\right)\left(\bar{\partial}^{(\lambda-k)} \partial_{-}^{k} \Phi \partial_{-}^{(\lambda-k)} \bar{\partial}^{k} \Phi\right) \\
& =-\frac{4}{3} g \frac{1}{\partial_{-}^{2 \lambda}} \bar{\Phi} \sum_{k=0}^{\lambda}(-1)^{k}\left[\left(\begin{array}{c}
\lambda-1 \\
k
\end{array}\right)+\left(\begin{array}{c}
\lambda-1 \\
k-1
\end{array}\right)\right]\left(\bar{\partial}^{(\lambda-k)} \partial_{-}^{k} \Phi \partial_{-}^{(\lambda-k)} \bar{\partial}^{k} \Phi\right) \\
& =-\frac{4}{3} g \frac{1}{\partial_{-}^{2 \lambda}} \bar{\Phi} \sum_{k=0}^{\lambda}(-1)^{k}\left(\begin{array}{c}
\lambda \\
k
\end{array}\right)\left(\bar{\partial}^{(\lambda-k)} \partial_{-}^{k} \Phi \partial_{-}^{(\lambda-k)} \bar{\partial}^{k} \Phi\right),
\end{aligned}
$$

matching the structures in (3.14) and (3.15) confirming that these are quadratic forms.

\section{Amplitude structures in higher spin theories}

We now examine the scattering amplitude structures that appear in the cubic Hamiltonians discussed thus far. Any four-vector can be expressed as a bispinor using the Pauli matrices, $p_{a \dot{a}}=p_{\mu} \sigma_{a \dot{a}}^{\mu}$, with $\operatorname{det}\left(p_{a \dot{a}}\right)$ yielding $-p^{\mu} p_{\mu}$. We introduce the spinor product

$$
\langle k l\rangle \equiv \sqrt{2} \frac{\left(k l_{-}-l k_{-}\right)}{\sqrt{k_{-} l_{-}}} .
$$

\subsection{The non-supersymmetric case}

Scattering amplitudes in non-supersymmetric higher spin theories, to first order in the coupling, were discussed in $[19,20]$. The main result, in momentum space, is that the cubic vertices in (2.7) and (2.8) may be obtained by raising the cubic vertex in pure YangMills theory (the $\lambda=1$ case), to the appropriate power [21, 22].

$$
L_{3}^{\lambda}=\left[\frac{\langle k l\rangle^{3}}{\langle l p\rangle\langle p k\rangle}\right]^{\lambda}=\left[L_{3}^{\mathrm{YM}}\right]^{\lambda} .
$$

\subsection{The supersymmetric case}

We turn now to the third new result in this paper, pertaining to scattering amplitude structures in maximally supersymmetric higher spin theories. The action corresponding to $(3.14)$, for even $\lambda$, is

$$
\begin{aligned}
S= & \int \mathrm{d}^{4} x \mathrm{~d}^{N} \theta \mathrm{d}^{N} \bar{\theta}\left\{\frac{1}{4} \bar{\Phi} \frac{\square}{\partial_{-}^{N / 2}} \Phi\right. \\
& \left.+\frac{1}{3} g\left(\frac{1}{\partial_{-}^{N / 2}} \Phi \sum_{n=0}^{\lambda}(-1)^{n}\left(\begin{array}{l}
\lambda \\
n
\end{array}\right)\left[\partial^{\lambda-n} \partial_{-}^{n} \bar{\Phi} \partial^{n} \partial_{-}^{\lambda-n} \bar{\Phi}\right]+\text { c.c. }\right)+\mathcal{O}\left(g^{2}\right)\right\},
\end{aligned}
$$

while that corresponding to (3.15) for odd $\lambda$ reads

$$
\begin{aligned}
S= & \int \mathrm{d}^{4} x \mathrm{~d}^{N} \theta \mathrm{d}^{N} \bar{\theta}\left\{\frac{1}{4} \bar{\Phi}^{a} \frac{\square}{\partial_{-}^{N / 2}} \Phi^{a}\right. \\
& \left.+\frac{1}{3} g f^{a b c}\left[\frac{1}{\partial_{-}^{N / 2}} \Phi^{a} \sum_{n=0}^{\lambda}(-1)^{n}\left(\begin{array}{l}
\lambda \\
n
\end{array}\right)\left[\partial^{\lambda-n} \partial_{-}^{n} \bar{\Phi}^{b} \partial^{n} \partial_{-}^{\lambda-n} \bar{\Phi}^{c}\right]+\text { c.c. }\right]+\mathcal{O}\left(g^{2}\right)\right\},
\end{aligned}
$$


with $N=4 \lambda$. In momentum space, both cubic vertices have the following basic structure (measure and constants suppressed)

$$
\begin{aligned}
& \frac{\delta^{4}(p+k+l)}{\left(k_{-}+l_{-}\right)^{2 \lambda}} \sum_{n=0}^{\lambda}(-1)^{n}\left(\begin{array}{l}
\lambda \\
n
\end{array}\right)\left[k^{\lambda-n} k_{-}^{n} l^{n} l_{-}^{\lambda-n}\right] \tilde{\Phi}(p) \tilde{\bar{\Phi}}(k) \tilde{\bar{\Phi}}(l)+c . c . \\
& \quad=\frac{\delta^{4}(p+k+l)}{\left(k_{-}+l_{-}\right)^{2 \lambda}} \sum_{n=0}^{\lambda}(-1)^{n}\left(\begin{array}{l}
\lambda \\
n
\end{array}\right)\left[\left(k l_{-}\right)^{\lambda-n}\left(k_{-} l\right)^{n}\right] \tilde{\Phi}(p) \tilde{\bar{\Phi}}(k) \tilde{\bar{\Phi}}(l)+c . c . \\
& \quad=\delta^{4}(p+k+l) \frac{\left(k l_{-}-k_{-} l\right)^{\lambda}}{\left(k_{-}+l_{-}\right)^{2 \lambda}} \tilde{\Phi}(p) \tilde{\bar{\Phi}}(k) \tilde{\bar{\Phi}}(l)
\end{aligned}
$$

The momentum conserving delta function $\delta^{4}(p+k+l)$ implies that

$$
\begin{aligned}
\langle l p\rangle & =\sqrt{\frac{2}{p_{-} l_{-}}}\left(k l_{-}-l k_{-}\right)=\frac{\sqrt{k_{-}}}{\sqrt{-\left(k_{-}+l_{-}\right)}}\langle k l\rangle \\
\langle p k\rangle & =\sqrt{\frac{2}{p_{-} k_{-}}}\left(k l_{-}-l k_{-}\right)=\frac{\sqrt{l_{-}}}{\sqrt{-\left(k_{-}+l_{-}\right)}}\langle k l\rangle .
\end{aligned}
$$

The cubic vertex is then

$$
\begin{aligned}
\frac{\left(k l_{-}-k_{-} l\right)^{\lambda}}{\left(k_{-}+l_{-}\right)^{2 \lambda}} & =\left[\left(\frac{k l_{-}-k_{-} l}{k_{-} l_{-}}\right)\left(k_{-}+l_{-}\right)\right]^{\lambda} \frac{\left(k_{-} l_{-}\right)^{\lambda}}{\left(k_{-}+l_{-}\right)^{3 \lambda}} \\
& =\left[\frac{\langle k l\rangle^{3}}{\langle l p\rangle\langle p k\rangle}\right]^{\lambda} \frac{\left(k_{-} l_{-}\right)^{\lambda}}{\left(k_{-}+l_{-}\right)^{3 \lambda}} \\
& =\left[\frac{\langle k l\rangle^{3}}{\langle l p\rangle\langle p k\rangle} \frac{k_{-} l_{-}}{\left(k_{-}+l_{-}\right)^{3}}\right]^{\lambda}=L_{3 \text { susy }}^{\lambda}
\end{aligned}
$$

The light-cone cubic vertex for $\mathcal{N}=4$ superYang-Mills was previously shown to be [23]

$$
L_{3}^{\mathcal{N}=4}=\left[\frac{\langle k l\rangle^{3}}{\langle l p\rangle\langle p k\rangle} \frac{k_{-} l_{-}}{\left(k_{-}+l_{-}\right)^{3}}\right] .
$$

Therefore the coefficient of the cubic vertex in maximally supersymmetric higher spin theories is equal to the corresponding vertex in the $\mathcal{N}=4$ theory, raised to the power $\lambda$.

$$
L_{3 \text { susy }}^{\lambda}=\left[\frac{\langle k l\rangle^{3}}{\langle l p\rangle\langle p k\rangle} \frac{k_{-} l_{-}}{\left(k_{-}+l_{-}\right)^{3}}\right]^{\lambda}=\left[L_{3}^{\mathcal{N}=4}\right]^{\lambda} .
$$

Assuming that light-cone higher spin quartic vertices exist and can be written down in this non-covariant gauge, it is likely that the structural relationship in (4.9) will hold at higher orders as well, suggesting the existence of KLT-like relations [24-26].

It is surprising that the light-cone Hamiltonians describing Yang-Mills, gravity and higher spin fields all exhibit this quadratic form structure. ${ }^{3}$ The light-cone Hamiltonian very likely remains a quadratic form with the $\mathcal{W}$ picking up higher order corrections. That the form appears only in the pure and maximally supersymmetric versions seems rather

\footnotetext{
${ }^{3}$ The Hamiltonian for the BLG theory is also a quadratic form [27-29].
} 
striking. There are a number of issues to investigate including implications for higher spin symmetries, residual gauge invariance $[1,2]$ and particularly field configurations $\mathcal{W}=0$ with vanishing energy.

This quadratic form structure, at the level of the Hamiltonian, seems to suggest a change of variables from $\Phi$ to $\mathcal{W}$. However, moving to the Dirac-Feynman path integral, which generates the quantum action, is unlikely to be straightforward. This idea, of simplifying the theory using a change of variables, is reminiscent of the Nicolai map in Yang-Mills theories [30, 31]. Given the ubiquitous nature of $\mathcal{N}=4$ Yang-Mills theory, a connection between these quadratic form structures, the map [32] and integrability should prove extremely interesting.

\section{A Superfield identities}

The following identities based on binomial expansions are useful in our calculations [9]

$$
\begin{aligned}
\sum_{k=0}^{\lambda-1}(-1)^{k} & \left(\begin{array}{c}
\lambda-1 \\
k
\end{array}\right)\left(\bar{\partial}^{(\lambda-1-k)} \partial_{-}^{k} \Phi \partial_{-}^{(\lambda-k-1)} \bar{\partial}^{k} \Phi\right) \\
= & \sum_{k=0}^{\lambda-1}(-1)^{k}\left(\begin{array}{c}
\lambda-1 \\
k
\end{array}\right) \bar{\partial}^{(\lambda-1-k)} \partial_{-}{ }^{(k)}\left(\Phi \partial_{-}^{(\lambda-k-1)} \bar{\partial}^{k} \Phi\right)
\end{aligned}
$$

\section{Variant 1.}

$$
\begin{aligned}
\sum_{k=0}^{\lambda-1}(-1)^{k} & \left(\begin{array}{c}
\lambda-1 \\
k
\end{array}\right)\left(\bar{\partial}^{(\lambda-1-k)} \partial_{-}^{k} \Phi \partial_{-}^{(\lambda-k-1)} \bar{\partial}^{k} \theta^{1} \bar{d}_{1} \Phi\right) \\
& =\sum_{k=0}^{\lambda-1}(-1)^{k}\left(\begin{array}{c}
\lambda-1 \\
k
\end{array}\right) \bar{\partial}^{(\lambda-1-k)} \partial_{-}^{k}\left(\Phi \partial_{-}^{(\lambda-k-1)} \bar{\partial}^{k} \theta^{1} \bar{d}_{1} \Phi\right)
\end{aligned}
$$

This identity is adapted from (A.1), with the redefinitions $\Phi_{1} \equiv \Phi_{1}$ and $\theta^{1} \bar{d}_{1} \Phi_{2} \equiv \Phi_{2}$, allowed because the identity is purely combinatorial in nature.

\section{Variant 2.}

$$
\begin{aligned}
\sum_{k=0}^{\lambda-1}(-1)^{k} & \left(\begin{array}{c}
\lambda-1 \\
k
\end{array}\right)\left(\bar{\partial}^{(\lambda-1-k)} \partial_{-}^{k} \Phi \partial_{-}^{(\lambda-k-1)} \bar{\partial}^{(k+1)} \theta^{1} \bar{d}_{1} \Phi\right) \\
& =\sum_{k=0}^{\lambda-1}(-1)^{k}\left(\begin{array}{c}
\lambda-1 \\
k
\end{array}\right) \bar{\partial}^{(\lambda-1-k)} \partial_{-}^{k}\left(\Phi \partial_{-}^{(\lambda-k-1)} \bar{\partial}^{(k+1)} \theta^{1} \bar{d}_{1} \Phi\right)
\end{aligned}
$$

Again adapted from (A.1), with the redefinitions $\Phi_{1} \equiv \Phi_{1}$ and $\theta^{1} \bar{d}_{1} \bar{\partial} \Phi_{2} \equiv \Phi_{2}$.

Open Access. This article is distributed under the terms of the Creative Commons Attribution License (CC-BY 4.0), which permits any use, distribution and reproduction in any medium, provided the original author(s) and source are credited. 


\section{References}

[1] S. Ananth, L. Brink and M. Mali, Yang-Mills theories and quadratic forms, JHEP 08 (2015) 153 [arXiv: 1507.01068] [INSPIRE].

[2] S. Ananth, L. Brink, S. Majumdar, M. Mali and N. Shah, Gravitation and quadratic forms, JHEP 03 (2017) 169 [arXiv: 1702.06261] [INSPIRE].

[3] M. Henneaux, D. Persson and P. Spindel, Spacelike Singularities and Hidden Symmetries of Gravity, Living Rev. Rel. 11 (2008) 1 [arXiv:0710.1818] [InSPIRE].

[4] S. Ananth, L. Brink and S. Majumdar, A hidden symmetry in quantum gravity, JHEP 11 (2018) 078 [arXiv: 1808.02498] [INSPIRE].

[5] S. Ananth, L. Brink and S. Majumdar, $E_{8}$ in $\mathcal{N}=8$ supergravity in four dimensions, JHEP 01 (2018) 024 [arXiv: 1711.09110] [INSPIRE].

[6] S. Ananth, L. Brink and S. Majumdar, Exceptional versus superPoincaré algebra as the defining symmetry of maximal supergravity, JHEP 03 (2016) 051 [arXiv:1601.02836] [INSPIRE].

[7] A.K.H. Bengtsson, I. Bengtsson and L. Brink, Cubic Interaction Terms for Arbitrary Spin, Nucl. Phys. B 227 (1983) 31 [INSPIRE].

[8] A.K.H. Bengtsson, I. Bengtsson and N. Linden, Interacting Higher Spin Gauge Fields on the Light Front, Class. Quant. Grav. 4 (1987) 1333 [INSPIRE].

[9] A.K.H. Bengtsson, I. Bengtsson and L. Brink, Cubic Interaction Terms for Arbitrarily Extended Supermultiplets, Nucl. Phys. B 227 (1983) 41 [INSPIRE].

[10] H. Elvang and Y.-t. Huang, Scattering Amplitudes, arXiv:1308.1697 [INSPIRE].

[11] S. Mandelstam, Light Cone Superspace and the Ultraviolet Finiteness of the $N=4$ Model, Nucl. Phys. B 213 (1983) 149 [InSPIRE].

[12] R.R. Metsaev, Cubic interaction vertices of massive and massless higher spin fields, Nucl. Phys. B 759 (2006) 147 [hep-th/0512342] [INSPIRE].

[13] R.R. Metsaev, Cubic interaction vertices for fermionic and bosonic arbitrary spin fields, Nucl. Phys. B 859 (2012) 13 [arXiv:0712.3526] [InSPIRE].

[14] A.K.H. Bengtsson, Systematics of Higher-spin Light-front Interactions, arXiv:1205.6117 [INSPIRE].

[15] A.K.H. Bengtsson, Notes on Cubic and Quartic Light-Front Kinematics, arXiv: 1604.01974 [INSPIRE].

[16] S. Ananth, A. Kar, S. Majumdar and N. Shah, Deriving spin-1 quartic interaction vertices from closure of the Poincaré algebra, Nucl. Phys. B 926 (2018) 11 [arXiv:1707.05871] [INSPIRE].

[17] S. Ananth, L. Brink, S.-S. Kim and P. Ramond, Non-linear realization of PSU $(2,2 \mid 4)$ on the Light-Cone, Nucl. Phys. B 722 (2005) 166 [hep-th/0505234] [INSPIRE].

[18] S. Ananth, L. Brink, R. Heise and H.G. Svendsen, The $N=8$ Supergravity Hamiltonian as a Quadratic Form, Nucl. Phys. B 753 (2006) 195 [hep-th/0607019] [INSPIRE].

[19] S. Ananth, Spinor helicity structures in higher spin theories, JHEP 11 (2012) 089 [arXiv: 1209.4960] [INSPIRE]. 
[20] Y.S. Akshay and S. Ananth, Cubic interaction vertices in higher spin theories, J. Phys. A 47 (2014) 045401 [arXiv: 1304.8082] [INSPIRE].

[21] Y.S. Akshay and S. Ananth, Factorization of cubic vertices involving three different higher spin fields, Nucl. Phys. B 887 (2014) 168 [arXiv: 1404.2448] [INSPIRE].

[22] Y.S. Akshay and S. Ananth, Fermi-Bose cubic couplings in light-cone field theories, Phys. Rev. D 91 (2015) 085029 [arXiv: 1504.00967] [INSPIRE].

[23] S. Ananth, S. Kovacs and S. Parikh, A manifestly MHV Lagrangian for $N=4$ Yang-Mills, JHEP 05 (2011) 051 [arXiv: 1101.3540] [INSPIRE].

[24] H. Kawai, D.C. Lewellen and S.H.H. Tye, A Relation Between Tree Amplitudes of Closed and Open Strings, Nucl. Phys. B 269 (1986) 1 [InSPIRE].

[25] S. Ananth and S. Theisen, KLT relations from the Einstein-Hilbert Lagrangian, Phys. Lett. B 652 (2007) 128 [arXiv:0706.1778] [INSPIRE].

[26] S. Ananth, Gravity and Yang-Mills theory, Int. J. Mod. Phys. D 19 (2010) 2379 [arXiv: 1011.3287] [INSPIRE].

[27] J. Bagger and N. Lambert, Gauge symmetry and supersymmetry of multiple M2-branes, Phys. Rev. D $\mathbf{7 7}$ (2008) 065008 [arXiv: 0711.0955] [INSPIRE].

[28] A. Gustavsson, Algebraic structures on parallel M2-branes, Nucl. Phys. B 811 (2009) 66 [arXiv:0709.1260] [INSPIRE].

[29] D. Belyaev, L. Brink, S.-S. Kim and P. Ramond, The BLG Theory in Light-Cone Superspace, JHEP 04 (2010) 026 [arXiv: 1001.2001] [INSPIRE].

[30] H. Nicolai, Supersymmetry and Functional Integration Measures, Nucl. Phys. B 176 (1980) 419 [INSPIRE].

[31] K. Dietz and O. Lechtenfeld, Ghost-free quantization of non-abelian gauge theories via the Nicolai transformation of their supersymmetric extensions, Nucl. Phys. B 259 (1985) 397 [INSPIRE].

[32] S. Ananth, H. Nicolai, C. Pandey and S. Pant, Supersymmetric Yang-Mills theories:not quite the usual perspective, J. Phys. A 53 (2020) 17 [arXiv:2001.02768] [INSPIRE]. 